\title{
Robust stability analysis of quaternion-valued neural networks via LMI approach
}

\author{
Xiaofeng Chen ${ }^{1 *} \mathbb{D}$, Lianjie Li and Zhongshan $\mathrm{Li}^{2}$
}

"Correspondence: xxffch@126.com 'Department of Mathematics, Chongqing Jiaotong University, Chongqing, China

Full list of author information is available at the end of the article

\begin{abstract}
This paper is concerned with the issue of robust stability for quaternion-valued neural networks (QVNNs) with leakage, discrete and distributed delays by employing a linear matrix inequality (LMI) approach. Based on the homeomorphic mapping theorem, the quaternion matrix theorem and the Lyapunov theorem, some criteria are developed in the form of real-valued LMls for guaranteeing the existence, uniqueness, and global robust stability of the equilibrium point of the delayed QVNNs. Two numerical examples are provided to demonstrate the effectiveness of the obtained results.
\end{abstract}

Keywords: Quaternion-valued neural networks; Delay effects; Global robust stability; Linear matrix inequality; Modulus inequality technique

\section{Introduction}

The quaternions are members of a noncommutative division algebra invented independently by Carl Friedrich Gauss in 1819 and William Rowan Hamilton in 1843 [1]. Quaternions provide a concise mathematical method for representing the automorphisms of three- and four-dimensional spaces. The representations by quaternions are more compact and quicker to compute than the representations by matrices [2]. For this reason, an increasing number of applications based on quaternions are found in various fields, such as computer graphics, quantum mechanics, attitude control, signal processing, and orbital mechanics [3-5]. For example, in attitude-control systems, it will lead to the problem of the so-called "gimbal lock" by using Euler angles. As an alternative approach, quaterions have the technical advantage not to suffer from the problem [6].

On the other hand, over the past three decades, neural networks (NNs) have been applied in various areas throughout science and engineering, such as signal processing, image processing, pattern recognition, associative memory and optimization [7-16]. Furthermore, real-valued NNs (RVNNs) and complex-valued NNs (CVNNs) have been extensively investigated and a great number of results have been reported [17-22]. Recently, quaternion-valued neural networks (QVNNs) have drawn a great deal of attention [23, 24]. Due to the simple representation of quaternions and the high efficiency in dealing with multidimensional data, QVNNs have demonstrated better performances than CVNNs and RVNNs in their wide applications [25-31]. For example, in the image compression

(c) The Author(s) 2018. This article is distributed under the terms of the Creative Commons Attribution 4.0 International License (http://creativecommons.org/licenses/by/4.0/), which permits unrestricted use, distribution, and reproduction in any medium, provided you give appropriate credit to the original author(s) and the source, provide a link to the Creative Commons license, and indicate if changes were made. 
$[25,26]$, one color is synthesized by the three primary colors with a certain proportion, which needs three real- or complex-valued neurons to store. However, one quaternionvalued neuron is enough to represent one color via three channels $l, j$ and $\kappa$ of QVNNs, which leads to a significant reduction in the dimension of the system and to a great increase in the computational efficiency. In some practical applications, it is required that the designed system has a unique equilibrium point which is globally stable. Therefore, the dynamics of QVNNs has been an active research topic [32-36]. In [32, 33], some $\mu$ stability criteria in the form of linear matrix inequalities (LMIs) were provided for QVNNs with time-varying delays. In [34], several sufficient conditions were presented to check the global exponential stability for QVNNs with time-varying delays. In [35], several sufficient criteria were derived to ensure the existence, uniqueness, and global robust stability of the equilibrium point for delayed QVNNs with parameter uncertainties. In [36], some algebraic conditions on the global dissipativity for QVNNs with time-varying delays were devised.

Moreover, when implementing neural networks, time delays are unavoidably encountered due to delay transmission line, partial element equivalent circuit, integration and communication. The existence of time delays in neural networks frequently will lead to undesirable complex dynamical behaviors [37-39]. As pointed out by Gopalsamy [40], time delays in the negative feedback terms will have a tendency to destabilize a system, which are known as leakage or forgetting delays. Moreover, many biological and artificial neural networks contain inherent discrete time delays in signal transmission, which may cause oscillation and instability. Furthermore, since the neural networks usually are of a spatial nature associated with the presence of an amount of parallel pathway of a variety of axon sizes and lengths, it is desirable to model them by introducing distributed delays. In $[40,41]$, the stability problem was investigated for RVNNs with the introduction of the leakage delays. In $[42,43]$, some dynamical behaviors of RVNNs with distributed delays were studied. In [44], the multistability issue of competitive RVNNs with discrete and distributed delays was investigated. In $[45,46]$, the authors considered the effects of leakage and discrete delays in CVNNs.

Strongly motivated by the above discussions, in the present paper we consider the robust stability problem of QVNNs with leakage, discrete and distributed delays. There are two main challenging problems of the current research. The first one is how to construct a proper Lyapunov-Krasovskii functional corresponding to the considered QVNNs. The second one is how to make sure that the obtained criteria are depend on the upper and lower bounds of system parameters. For the first one, we adopt quaternion self-conjugate and positive definite matrices to construct the Lyapunov-Krasovskii functional so that we can directly deal with the QVNNs rather than any decomposition. For the second one, we utilize modulus inequality technique to compute the derivative of the LyapunovKrasovskii functional so that the obtained criteria are not only real-valued but also related to the bounds of parameters.

Notations Throughout this paper, $\mathbb{R}, \mathbb{C}$ and $\mathbb{H}$ denote the real field, the complex field and the skew field of quaternions, respectively. $\mathbb{R}^{n}, \mathbb{C}^{n}$ and $\mathbb{H}^{n}$ denote $n$-dimensional vectors with entries from $\mathbb{R}, \mathbb{C}$ and $\mathbb{H}$, respectively. $\mathbb{R}^{n \times m}, \mathbb{C}^{n \times m}$ and $\mathbb{H}^{n \times m}$ denote $n \times m$ matrices with entries from $\mathbb{R}, \mathbb{C}$ and $\mathbb{H}$, respectively. Specially, $\mathbb{R}_{d}^{n \times n}$ denotes $n \times n$ real diagonal matrices. The notation $\bar{A}, A^{T}$ and $A^{*}$ stand for the conjugate, the transpose 
and the conjugate transpose, respectively, of the matrix $A$. For $A=\left(a_{i j}\right)_{n \times n} \in \mathbb{C}^{n \times n}$, let $\|A\|=\sqrt{\sum_{i=1}^{n} \sum_{j=1}^{n}\left|a_{i j}\right|^{2}}$ denote the norm of $A$. The notation $X \geq Y$ (respectively, $X>Y$ ) means that $X-Y$ is positive semi-definite (respectively, positive definite). For a positive definite Hermitian matrix $P \in \mathbb{C}^{n \times n}, \lambda_{\max }(P)$ and $\lambda_{\min }(P)$ are defined as the largest and the smallest eigenvalues of $P$, respectively. In the four-dimensional algebra $\mathbb{H}$, the four basis elements are denoted by $1, l, J, \kappa$, which obey the following multiplication table: $\iota^{2}=\jmath^{2}=\kappa^{2}=-1, \imath \jmath=-\jmath \imath=\kappa, \jmath \kappa=-\kappa \jmath=\imath, \kappa \imath=-\imath \kappa=\jmath$, and $1 \cdot a=a \cdot 1=a$ for every quaternion $a$. For a quaternion $a=a_{0}+a_{1} l+a_{2} J+a_{3} \kappa \in \mathbb{H}$, we call $a_{0}, a_{1}$, $a_{2}$ and $a_{3}$ the first, second, third and fourth parts of the quaternion, respectively. Let $a^{*}=a_{0}-a_{1} l-a_{2} J-a_{3} \kappa$ be the conjugate of $a$, and $|a|=\sqrt{a_{0}^{2}+a_{1}^{2}+a_{2}^{2}+a_{3}^{2}}$ be the modulus of $a$. For $q=\left(q_{1}, q_{2}, \ldots, q_{n}\right)^{T} \in \mathbb{H}^{n}$, let $|q|=\left(\left|q_{1}\right|,\left|q_{2}\right|, \ldots,\left|q_{n}\right|\right)^{T}$ be the modulus of $q$, and $\|q\|=\sqrt{\sum_{i=1}^{n}\left|q_{i}\right|^{2}}$ be the norm of $q$. For $a, b \in \mathbb{H}, a \preceq b$ denotes $a_{i} \leq b_{i}, i=0,1,2,3$, where $a=a_{0}+a_{1} l+a_{2} J+a_{3} \kappa$ and $b=b_{0}+b_{1} l+b_{2} J+b_{3} \kappa$. For $A, B \in \mathbb{H}^{n \times n}, A \preceq B$ denotes $a_{i j} \preceq b_{i j}, i, j=1,2, \ldots, n$, where $A=\left(a_{i j}\right)_{n \times n}$ and $B=\left(b_{i j}\right)_{n \times n}$. In addition, the symbol $\star$ always denotes the conjugate transpose of a suitable block in a Hermitian matrix.

\section{Problem formulation and preliminaries}

Consider the following QVNNs model with three kinds of time delays including leakage delay, discrete delay and distributed delay:

$$
\dot{q}(t)=-D q(t-\delta)+A f(q(t))+B f(q(t-\tau))+C \int_{-\infty}^{t} k(t-s) f(q(s)) \mathrm{d} s+J,
$$

for $t \geq 0$, where $q(t)=\left(q_{1}(t), q_{2}(t), \ldots, q_{n}(t)\right)^{T} \in \mathbb{H}^{n}$ is the state vector of the neural networks with $n$ neurons at time $t . D=\operatorname{diag}\left(d_{1}, d_{2}, \ldots, d_{n}\right) \in \mathbb{R}_{d}^{n \times n}$ where $d_{i}>0$ $(i=1,2, \ldots, n)$ is the self-feedback connection weight matrix; $A=\left(a_{i j}\right)_{n \times n} \in \mathbb{H}^{n \times n}, B=$ $\left(b_{i j}\right)_{n \times n} \in \mathbb{H}^{n \times n}$ and $C=\left(c_{i j}\right)_{n \times n} \in \mathbb{H}^{n \times n}$ are, respectively, the connection weight matrix, the discretely delayed connection weight matrix and the distributively delayed connection weight matrix. $J=\left(J_{1}, J_{2}, \ldots, J_{n}\right)^{T} \in \mathbb{H}^{n}$ is the external input vector. $f(q(t))=$ $\left(f_{1}\left(q_{1}(t)\right), f_{2}\left(q_{2}(t)\right), \ldots, f_{n}\left(q_{n}(t)\right)\right)^{T} \in \mathbb{H}^{n}$ denotes the neuron activations. $\delta>0$ and $\tau>0$ are the leakage time delay and the discrete time delay, respectively. $k(\cdot):[0,+\infty) \rightarrow[0,+\infty)$ is the delay kernel, which satisfies $\int_{0}^{\infty} k(s) \mathrm{d} s=1$.

The following assumptions will be needed throughout the paper:

$\left(\mathrm{A}_{1}\right)$ The parameters $D, A, B, C$, $J$ in QVNNs (1) are assumed to be in the following sets, respectively,

$$
\begin{aligned}
& D_{I}=\left\{D \in \mathbb{R}_{d}^{n \times n}: 0<\check{D} \preceq D \preceq \hat{D}\right\}, \\
& A_{I}=\left\{A \in \mathbb{H}^{n \times n}: \check{A} \preceq A \preceq \hat{A}\right\}, \\
& B_{I}=\left\{B \in \mathbb{H}^{n \times n}: \check{B} \preceq B \preceq \hat{B}\right\}, \\
& C_{I}=\left\{C \in \mathbb{H}^{n \times n}: \check{C} \preceq C \preceq \hat{C}\right\}, \\
& J_{I}=\left\{J \in \mathbb{H}^{n}: \check{J} \preceq J \preceq \hat{J}\right\},
\end{aligned}
$$

where $\check{D}, \hat{D} \in \mathbb{R}_{d}^{n \times n}, \check{A}, \hat{A}, \check{B}, \hat{B}, \check{C}, \hat{C} \in \mathbb{H}^{n \times n}$, and $\check{J}, \hat{J} \in \mathbb{H}^{n}$. Moreover, let $\check{A}=\left(\check{a}_{i j}\right)_{n \times n}$, $\hat{A}=\left(\hat{a}_{i j}\right)_{n \times n}, \check{B}=\left(\check{b}_{i j}\right)_{n \times n}, \hat{B}=\left(\hat{b}_{i j}\right)_{n \times n}, \check{C}=\left(\check{c}_{i j}\right)_{n \times n}$, and $\hat{C}=\left(\hat{c}_{i j}\right)_{n \times n}$. Then we de- 
fine $\tilde{A}=\left(\tilde{a}_{i j}\right)_{n \times n}, \tilde{B}=\left(\tilde{b}_{i j}\right)_{n \times n}$ and $\tilde{C}=\left(\tilde{c}_{i j}\right)_{n \times n}$, where $\tilde{a}_{i j}=\max \left\{\left|\tilde{a}_{i j}\right|,\left|\hat{a}_{i j}\right|\right\}, \tilde{b}_{i j}=$ $\max \left\{\left|\check{b}_{i j}\right|,\left|\hat{b}_{i j}\right|\right\}$ and $\tilde{c}_{i j}=\max \left\{\left|\check{c}_{i j}\right|,\left|\hat{c}_{i j}\right|\right\}$.

$\left(\mathrm{A}_{2}\right)$ For $i=1,2, \ldots, n$, the neuron activation function $f_{i}$ is continuous and satisfies

$$
\left|f_{i}\left(s_{1}\right)-f_{i}\left(s_{2}\right)\right| \leq \gamma_{i}\left|s_{1}-s_{2}\right|, \quad \forall s_{1}, s_{2} \in \mathbb{H},
$$

where $\gamma_{i}$ is a real constant. Moreover, define $\Gamma=\operatorname{diag}\left(\gamma_{1}, \gamma_{2}, \ldots, \gamma_{n}\right)$.

Definition 1 The QVNNs defined by (1) with the parameter ranges defined by $\left(\mathrm{A}_{1}\right)$ are globally asymptotically robust stable if the unique equilibrium point $\check{q}$ of QVNNs (1) is globally asymptotically stable for all $D \in D_{I}, A \in A_{I}, B \in B_{I}, C \in C_{I}$ and $J \in J_{I}$.

Lemma 1 ([35]) For any $x, y \in \mathbb{H}^{n}$, if $P \in \mathbb{H}^{n \times n}$ is a positive definite Hermitian matrix, then

$$
x^{*} y+y^{*} x \leq x^{*} P x+y^{*} P^{-1} y .
$$

Lemma 2 ([35]) If $H(z): \mathbb{H}^{n} \rightarrow \mathbb{H}^{n}$ is a continuous map and satisfies the following conditions:

(i) $H(z)$ is injective on $\mathbb{H}^{n}$,

(ii) $\lim _{\|z\| \rightarrow \infty}\|H(z)\|=\infty$,

then $H(z)$ is a homeomorphism of $\mathbb{H}^{n}$ onto itself.

Lemma 3 ([35]) For any positive definite constant Hermitian matrix $W \in \mathbb{H}^{n \times n}$ and any scalar function $\omega(s):[a, b] \rightarrow \mathbb{H}^{n}$ with scalars $a<b$ such that the integrations concerned are well defined,

$$
\left(\int_{a}^{b} \omega(s) \mathrm{d} s\right)^{*} W\left(\int_{a}^{b} \omega(s) \mathrm{d} s\right) \leq(b-a) \int_{a}^{b} \omega^{*}(s) W \omega(s) \mathrm{d} s .
$$

In the following, we provide some modulus inequalities of quaternions, which play a major role in analyzing the problem in this paper.

Lemma 4 Suppose $A \in \mathbb{H}^{n \times n}, \check{A}=\left(\check{a}_{i j}\right)_{n \times n} \in \mathbb{H}^{n \times n}, \hat{A}=\left(\hat{a}_{i j}\right)_{n \times n} \in \mathbb{H}^{n \times n}$, and $\check{A} \preceq A \preceq \hat{A}$. Then, for any $x, y \in \mathbb{H}^{n}$, the following inequalities hold:

$$
\begin{aligned}
& x^{*} A^{*} A x \leq|x|^{T}|A|^{T}|A||x| \leq|x|^{T} \tilde{A}^{T} \tilde{A}|x|, \\
& x^{*} A^{*} y+y^{*} A x \leq|x|^{T}|A|^{T}|y|+|y|^{T}|A||x| \leq|x|^{T} \tilde{A}^{T}|y|+|y|^{T} \tilde{A}|x|,
\end{aligned}
$$

where $\tilde{A}=\left(\tilde{a}_{i j}\right)_{n \times n}, \tilde{a}_{i j}=\max \left\{\left|\check{a}_{i j}\right|,\left|\hat{a}_{i j}\right|\right\}$.

Proof It should be noted that $|a+b| \leq|a|+|b|$ for any $a, b \in \mathbb{H}$. By the Cauchy-Schwarz inequality, the modulus inequalities (2) and (3) can be obtained. We omit the details because the proof is direct.

Remark 1 In Lemma 4, if $A$ is a real positive diagonal matrix, then $|A|=A$ and $\tilde{A}=\hat{A}$. Therefore, the modulus inequality (3) will reduce to

$$
x^{*} A^{*} y+y^{*} A x \leq|x|^{T} A^{T}|y|+|y|^{T} A|x| \leq|x|^{T} \hat{A}^{T}|y|+|y|^{T} \hat{A}|x| .
$$


Lemma 5 (Schur complement [47]) A given real symmetric matrix

$$
S=\left(\begin{array}{ll}
S_{11} & S_{12} \\
S_{21} & S_{22}
\end{array}\right),
$$

where $S_{11}^{T}=S_{11}, S_{12}^{T}=S_{21}$ and $S_{22}^{T}=S_{22}$, satisfies $S<0$ if and only if any one of the following two conditions holds:

(i) $S_{22}<0$ and $S_{11}-S_{12} S_{22}^{-1} S_{21}<0$,

(ii) $S_{11}<0$ and $S_{22}-S_{21} S_{11}^{-1} S_{12}<0$.

\section{Main results}

In this section, we first analyze the existence and uniqueness of the equilibrium point of the delayed QVNNs under Assumptions $\left(\mathrm{A}_{1}\right)$ and $\left(\mathrm{A}_{2}\right)$. Then we investigate the global robust stability of the equilibrium point of the delayed QVNNs.

Theorem 1 Under Assumptions $\left(\mathrm{A}_{1}\right)$ and $\left(\mathrm{A}_{2}\right)$, QVNNs (1) have a unique equilibrium point, if there exist four real positive diagonal matrices $U, V_{1}, V_{2}$ and $V_{3}$ such that the following LMI holds:

$$
\Sigma=\left(\begin{array}{cccc}
\Sigma_{11} & U \tilde{A} & U \tilde{B} & U \tilde{C} \\
\star & -V_{1} & 0 & 0 \\
\star & \star & -V_{2} & 0 \\
\star & \star & \star & -V_{3}
\end{array}\right)<0
$$

where $\Sigma_{11}=-\check{D} U-U \check{D}+\Gamma V_{1} \Gamma+\Gamma V_{2} \Gamma+\Gamma V_{3} \Gamma$.

Proof We define the following continuous map $\mathcal{H}: \mathbb{H}^{n} \rightarrow \mathbb{H}^{n}$ associated with system (1):

$$
\mathcal{H}(q)=-D z+A f(q)+B f(q)+C f(q)+J
$$

Then the proof is divided into two steps.

First, we prove that $\mathcal{H}(q)$ is an injective map on $\mathbb{H}^{n}$. Suppose that there exist $q, \tilde{q} \in \mathbb{H}^{n}$ with $q \neq \tilde{q}$, such that $\mathcal{H}(q)=\mathcal{H}(\tilde{q}) \mathrm{k}$, which implies that

$$
\begin{aligned}
0= & (q-\tilde{q})^{*} U[\mathcal{H}(q)-\mathcal{H}(\tilde{q})]+[\mathcal{H}(q)-\mathcal{H}(\tilde{q})]^{*} U(q-\tilde{q}) \\
= & -(q-\tilde{q})^{*}(U D+D U)(q-\tilde{q})+(q-\tilde{q})^{*} U A(f(q)-f(\tilde{q})) \\
& +(f(q)-f(\tilde{q}))^{*} A^{*} U(q-\tilde{q})+(q-\tilde{q})^{*} U B(f(q)-f(\tilde{q})) \\
& +(f(q)-f(\tilde{q}))^{*} B^{*} U(q-\tilde{q})+(q-\tilde{q})^{*} U C(f(q)-f(\tilde{q})) \\
& +(f(q)-f(\tilde{q}))^{*} C^{*} U(q-\tilde{q}) \\
\leq & -(q-\tilde{q})^{*}(U D+D U)(q-\tilde{q})+(q-\tilde{q})^{*} U(A) V_{1}^{-1} A^{*} U(q-\tilde{q}) \\
& +(f(q)-f(\tilde{q}))^{*} V_{1}(f(q)-f(\tilde{q}))+(q-\tilde{q})^{*} U B V_{2}^{-1} B^{*} U(q-\tilde{q}) \\
& +(f(q)-f(\tilde{q}))^{*} V_{2}(f(q)-f(\tilde{q}))+(q-\tilde{q})^{*} U C V_{3}^{-1} C^{*} U(q-\tilde{q}) \\
& +(f(q)-f(\tilde{q}))^{*} V_{3}(f(q)-f(\tilde{q}))
\end{aligned}
$$




$$
\begin{aligned}
\leq & |q-\tilde{q}|^{*}\left[-\check{D} U-U \check{D}+U \tilde{A} V_{1}^{-1} \tilde{A}^{*} U+U \tilde{B} V_{2}^{-1} \tilde{B}^{*} U\right. \\
& \left.+U \tilde{C} V_{3}^{-1} \tilde{C}^{*} U\right]|q-\tilde{q}|+(f(q)-f(\tilde{q}))^{*}\left(V_{1}+V_{2}+V_{3}\right)(f(q)-f(\tilde{q})) .
\end{aligned}
$$

Here, in the computation above, we have applied Lemmas 1 and 4 . Since $V_{1}, V_{2}$, and $V_{3}$ are real positive diagonal matrices, it follows from Assumption $\left(\mathrm{A}_{2}\right)$ that

$$
\begin{aligned}
& (f(q)-f(\tilde{q}))^{*}\left(V_{1}+V_{2}+V_{3}\right)(f(q)-f(\tilde{q})) \\
& \quad \leq(q-\tilde{q})^{*} \Gamma\left(V_{1}+V_{2}+V_{3}\right) \Gamma(q-\tilde{q})=|q-\tilde{q}|^{*} \Gamma\left(V_{1}+V_{2}+V_{3}\right) \Gamma|q-\tilde{q}| .
\end{aligned}
$$

We can get from (6) and (7)

$$
0 \leq|q-\tilde{q}|^{*} \Xi|q-\tilde{q}|
$$

where $\Xi=-\check{D} U-U \check{D}+\Gamma\left(V_{1}+V_{2}+V_{3}\right) \Gamma+U \tilde{A} V_{1}^{-1} \tilde{A}^{*} U+U \tilde{B} V_{2}^{-1} \tilde{B}^{*} U+U \tilde{C} V_{3}^{-1} \tilde{C}^{*} U$. From Lemma 5 and the LMI (4), we see that $\Xi<0$. Then $q-\tilde{q}=0$ from (8). Therefore, $\mathcal{H}(q)$ is an injective map on $\mathbb{H}^{n}$. The first step is completed.

Second, we prove $\|\mathcal{H}(q)\| \rightarrow \infty$ as $\|q\| \rightarrow \infty$. Let $\widetilde{\mathcal{H}}(q)=\mathcal{H}(q)-\mathcal{H}(0)$. By Lemmas 1 and 4 , we can compute that

$$
\begin{aligned}
q^{*} U \tilde{\mathcal{H}}(q)+\widetilde{\mathcal{H}}(q)^{*} U q= & -q^{*}(U D+D U) q+q^{*} U(A+B+C)(f(q)-f(0)) \\
& +(f(q)-f(0))^{*}\left(A^{*}+B^{*}+C^{*}\right) U q \\
\leq & -q^{*}(U D+D U) q+q^{*} U A V_{1}^{-1} A^{*} U q \\
& +q^{*} U B V_{2}^{-1} B^{*} U q+q^{*} U C V_{3}^{-1} C^{*} U q \\
& +(f(q)-f(0))^{*}\left(V_{1}+V_{2}+V_{3}\right)(f(q)-f(0)) \\
\leq & |q|^{*} \Xi|q| \leq-\lambda_{\min }(-\Xi)\|q\|^{2} .
\end{aligned}
$$

An application of the Cauchy-Schwarz inequality yields

$$
\lambda_{\min }(-\Xi)\|q\|^{2} \leq 2\left\|q^{*}\right\|\|U\|\|\tilde{\mathcal{H}}(q)\| .
$$

When $q \neq 0$, we have

$$
\|\tilde{\mathcal{H}}(q)\| \geq \frac{\lambda_{\min }(-\Xi)\|q\|}{2\|U\|} .
$$

Therefore, $\|\tilde{\mathcal{H}}(q)\| \rightarrow \infty$ as $\|q\| \rightarrow \infty$, which implies $\|\mathcal{H}(q)\| \rightarrow \infty$ as $\|q\| \rightarrow \infty$. From Lemma 2 we know that $\mathcal{H}(q)$ is a homeomorphism of $\mathbb{H}^{n}$. Thus, system (1) has a unique equilibrium point.

In what follows, we further consider the global robust stability of the equilibrium point based on Theorem 1.

Theorem 2 Under Assumptions $\left(\mathrm{A}_{1}\right)$ and $\left(\mathrm{A}_{2}\right)$, QVNNs (1) have a unique equilibrium point and the equilibrium point is globally robust stable, if there exist nine real positive 
diagonal matrices $P_{1}, P_{2}, P_{3}, P_{4}, Q_{1}, Q_{2}, R, S_{1}$ and $S_{2}$ such that the following $L M I$ holds:

$$
\Omega=\left(\begin{array}{cccccccc}
\Omega_{11} & 0 & 0 & 0 & P_{1} \tilde{A} & P_{1} \tilde{B} & P_{1} \tilde{C} & \hat{D} P_{1} \hat{D} \\
\star & \Omega_{22} & \Omega_{23} & 0 & Q_{1}^{T} \tilde{A} & Q_{1}^{T} \tilde{B} & Q_{1}^{T} \tilde{C} & 0 \\
\star & \star & \Omega_{33} & 0 & Q_{2}^{T} \tilde{A} & Q_{2}^{T} \tilde{B} & Q_{2}^{T} \tilde{C} & 0 \\
\star & \star & \star & \Omega_{44} & 0 & 0 & 0 & 0 \\
\star & \star & \star & \star & -S_{1} & 0 & 0 & \tilde{A}^{T} P_{1} \hat{D} \\
\star & \star & \star & \star & \star & -S_{2} & 0 & \tilde{B}^{T} P_{1} \hat{D} \\
\star & \star & \star & \star & \star & \star & -R & \tilde{C}^{T} P_{1} \hat{D} \\
\star & \star & \star & \star & \star & \star & \star & -P_{3}
\end{array}\right)<0
$$

where $\Omega_{11}=-P_{1} \check{D}-\check{D} P_{1}+P_{2}+\delta^{2} P_{3}+P_{4}+\Gamma R \Gamma+\Gamma S_{1} \Gamma, \Omega_{22}=-Q_{1}^{T}-Q_{1}, \Omega_{23}=Q_{1}^{T} \hat{D}+Q_{2}$, $\Omega_{33}=-P_{2}-Q_{2}^{T} \check{D}-\check{D} Q_{2}, \Omega_{44}=-P_{4}+\Gamma S_{2} \Gamma$.

Proof The proof will be divided into two steps. First, we will proof the QVNNs (1) have a unique equilibrium point under LMI (9) based on Theorem 1. Second, we will prove the equilibrium point is globally robust stable by the Lyapunov theorem.

Step 1: For convenience, we let

$$
\Omega_{1}=\left(\begin{array}{cccc}
\Omega_{11} & P_{1} \tilde{A} & P_{1} \tilde{B} & P_{1} \tilde{C} \\
\star & -S_{1} & 0 & 0 \\
\star & \star & -S_{2} & 0 \\
\star & \star & \star & -R
\end{array}\right), \quad \Omega_{2}=\left(\begin{array}{cccc}
\Theta & 0 & 0 & 0 \\
0 & 0 & 0 & 0 \\
0 & 0 & 0 & 0 \\
0 & 0 & 0 & 0
\end{array}\right)+\Omega_{1},
$$

where $\Theta=\Omega_{44}-P_{2}-\delta^{2} P_{3}$. It should be noted that $\Omega_{1}$ is a principal sub-matrix of $\Omega$ formed by rows $1,5,6,7$ and columns $1,5,6,7$. Therefore, $\Omega_{1}<0$. Since $\Theta<0$, we see that

$$
\Omega_{2}<0 .
$$

Then we let $U=P_{1}>0, V_{1}=S_{1}>0, V_{2}=S_{2}>0$ and $V_{3}=R>0$, we can obtain

$$
\Sigma=\Omega_{2}
$$

where $\Sigma$ is defined in LMI (4) of Theorem 1. It follows from (10) and (11) that $\Sigma<0$, which means LMI (4) holds. Thus, system (1) has a unique equilibrium point by Theorem 1.

Step 2: Let $\check{q}$ be the unique equilibrium point of system (1). For convenience, we shift the equilibrium to the origin by letting $\tilde{q}(t)=q(t)-\check{q}$, and then system (1) can be transformed into

$$
\dot{\tilde{q}}(t)=-D \tilde{q}(t-\delta)+A f(\tilde{q}(t))+B f(\tilde{q}(t-\tau))+C \int_{-\infty}^{t} k(t-s) f(\tilde{q}(s)) \mathrm{d} s,
$$

where $f(\tilde{q}(t))=f(q(t))-f(\check{q})$ and $f(\tilde{q}(t-\tau))=f(q(t-\tau))-f(\check{q})$.

With some preparation above, consider the following Lyapunov-Krasovskii functional:

$$
V(t)=V_{1}(t)+V_{2}(t)+V_{3}(t)+V_{4}(t)+V_{5}(t)
$$


Chen et al. Advances in Difference Equations (2018) 2018:131

Page 8 of 20

where

$$
\begin{aligned}
& V_{1}(t)=\left(\tilde{q}(t)-D \int_{t-\delta}^{t} \tilde{q}(s) \mathrm{d} s\right)^{*} P_{1}\left(\tilde{q}(t)-D \int_{t-\delta}^{t} \tilde{q}(s) \mathrm{d} s\right) \\
& V_{2}(t)=\int_{t-\delta}^{t} \tilde{q}^{*}(s) P_{2} \tilde{q}(s) \mathrm{d} s \\
& V_{3}(t)=\delta \int_{0}^{\delta} \int_{t-u}^{t} \tilde{q}^{*}(s) P_{3} \tilde{q}(s) \mathrm{d} s \mathrm{~d} u \\
& V_{4}(t)=\int_{t-\tau}^{t} \tilde{q}^{*}(s) P_{4} \tilde{q}(s) \mathrm{d} s \\
& V_{5}(t)=\sum_{j=1}^{n} r_{j} \int_{0}^{\infty} k(s) \int_{t-s}^{t} f_{j}^{*}\left(\tilde{q}_{j}(t)\right) f_{j}\left(\tilde{q}_{j}(t)\right) \mathrm{d} t \mathrm{~d} s,
\end{aligned}
$$

where $r_{j}$ are the main diagonal entries of $R$. That is, $R=\operatorname{diag}\left(r_{1}, r_{2}, \ldots, r_{n}\right)$.

Then the derivatives of $V_{1}(t), V_{2}(t), V_{3}(t), V_{4}(t)$ and $V_{5}(t)$ are calculated as follows:

$$
\begin{aligned}
& \dot{V}_{1}(t)=\left[\tilde{q}(t)-D \int_{t-\delta}^{t} \tilde{q}(s) \mathrm{d} s\right]^{*} P_{1}[\dot{\tilde{q}}(t)-D \tilde{q}(t)+D \tilde{q}(t-\delta)] \\
& +[\dot{\tilde{q}}(t)-D \tilde{q}(t)+D \tilde{q}(t-\delta)]^{*} P_{1}\left[\tilde{q}(t)-D \int_{t-\delta}^{t} \tilde{q}(s) \mathrm{d} s\right] \\
& =\left[\tilde{q}(t)-D \int_{t-\delta}^{t} \tilde{q}(s) \mathrm{d} s\right]^{*} P_{1}[-D \tilde{q}(t)+A f(\tilde{q}(t))+B f(\tilde{q}(t-\tau)) \\
& \left.+C \int_{-\infty}^{t} k(t-s) f(q(s)) \mathrm{d} s\right]+[-D \tilde{q}(t)+A f(\tilde{q}(t))+B f(\tilde{q}(t-\tau)) \\
& \left.+C \int_{-\infty}^{t} k(t-s) f(q(s)) \mathrm{d} s\right]^{*} P_{1}\left[\tilde{q}(t)-D \int_{t-\delta}^{t} \tilde{q}(s) \mathrm{d} s\right] \\
& =-\tilde{q}^{*}(t)\left(P_{1} D+D P_{1}\right) \tilde{q}(t)+\tilde{q}^{*}(t) P_{1} A f(\tilde{q}(t))+f^{*}(\tilde{q}(t)) A^{*} P_{1} \tilde{q}(t) \\
& +\tilde{q}^{*}(t) P_{1} B f(\tilde{q}(t-\tau))+f^{*}(\tilde{q}(t-\tau)) B^{*} P_{1} \tilde{q}(t) \\
& +\tilde{q}^{*}(t) P_{1} C\left[\int_{-\infty}^{t} k(t-s) f(q(s)) \mathrm{d} s\right]+\left[\int_{-\infty}^{t} k(t-s) f(q(s)) \mathrm{d} s\right]^{*} \\
& \times C^{*} P_{1} \tilde{q}(t)+\tilde{q}^{*}(t) D P_{1} D\left[\int_{t-\delta}^{t} \tilde{q}(s) \mathrm{d} s\right]+\left[\int_{t-\delta}^{t} \tilde{q}(s) \mathrm{d} s\right]^{*} D P_{1} D \tilde{q}(t) \\
& -f^{*}(\tilde{q}(t)) A^{*} P_{1} D\left[\int_{t-\delta}^{t} \tilde{q}(s) \mathrm{d} s\right]-\left[\int_{t-\delta}^{t} \tilde{q}(s) \mathrm{d} s\right]^{*} D P_{1} A f(\tilde{q}(t)) \\
& -f^{*}(\tilde{q}(t-\tau)) B^{*} P_{1} D\left[\int_{t-\delta}^{t} \tilde{q}(s) \mathrm{d} s\right] \\
& -\left[\int_{t-\delta}^{t} \tilde{q}(s) \mathrm{d} s\right]^{*} D P_{1} B f(\tilde{q}(t-\tau)) \\
& -\left[\int_{-\infty}^{t} k(t-s) f(q(s)) \mathrm{d} s\right]^{*} C^{*} P_{1} D\left[\int_{t-\delta}^{t} \tilde{q}(s) \mathrm{d} s\right] \\
& -\left[\int_{t-\delta}^{t} \tilde{q}(s) \mathrm{d} s\right]^{*} D P_{1} C\left[\int_{-\infty}^{t} k(t-s) f(q(s)) \mathrm{d} s\right] \text {, }
\end{aligned}
$$




$$
\begin{aligned}
\dot{V}_{2}(t)= & \tilde{q}^{*}(t) P_{2} \tilde{q}(t)-\tilde{q}^{*}(t-\delta) P_{2} \tilde{q}(t-\delta), \\
\dot{V}_{3}(t)= & \delta^{2} \tilde{q}^{*}(t) P_{3} \tilde{q}(t)-\delta \int_{0}^{\delta} \tilde{q}^{*}(t-u) P_{3} \tilde{q}(t-u) \mathrm{d} u \\
= & \delta^{2} \tilde{q}^{*}(t) P_{3} \tilde{q}(t)-\delta \int_{t-\delta}^{t} \tilde{q}^{*}(s) P_{3} \tilde{q}(s) \mathrm{d} s \\
\leq & \delta^{2} \tilde{q}^{*}(t) P_{3} \tilde{q}(t)-\left[\int_{t-\delta}^{t} \tilde{q}(s) \mathrm{d} s\right]^{*} P_{3}\left[\int_{t-\delta}^{t} \tilde{q}(s) \mathrm{d} s\right], \\
\dot{V}_{4}(t)= & \tilde{q}^{*}(t) P_{4} \tilde{q}(t)-\tilde{q}^{*}(t-\tau) P_{4} \tilde{q}(t-\tau), \\
\dot{V}_{5}(t)= & \sum_{j=1}^{n} r_{j} \int_{0}^{+\infty} k(s) f_{j}^{*}(\tilde{q}(t)) f_{j}(\tilde{q}(t)) \mathrm{d} s \\
& -\sum_{j=1}^{n} r_{j} \int_{0}^{+\infty} k(s) f_{j}^{*}(\tilde{q}(t-s)) f_{j}(\tilde{q}(t-s)) \mathrm{d} s \\
= & f^{*}(\tilde{q}(t)) R f(\tilde{q}(t)) \\
& -\sum_{j=1}^{n} r_{j} \int_{0}^{+\infty} k(s) \int_{0}^{+\infty} k(s) f_{j}^{*}(\tilde{q}(t-s)) f_{j}(\tilde{q}(t-s)) \mathrm{d} s \\
\leq & \tilde{q}^{*}(t) \Gamma R \Gamma \tilde{q}(t) \\
& -\sum_{j=1}^{n} r_{j} \int_{0}^{+\infty} k(s) f_{j}^{*}(\tilde{q}(t-s)) \mathrm{d} s \int_{0}^{+\infty} k(s) f_{j}(\tilde{q}(t-s)) \mathrm{d} s \\
\leq & \tilde{q}(t) \Gamma R \Gamma \tilde{q}(t) \\
& -\left[\int_{-\infty}^{t} k(t-s) f(q(s)) \mathrm{d} s\right]^{*} R\left[\int_{-\infty}^{t} k(t-s) f(q(s)) \mathrm{d} s\right] .
\end{aligned}
$$

In deriving inequality (20), we have made use of Lemma 3. Since $S_{1}$ and $S_{2}$ are real positive diagonal matrices, it follows from Assumption $\left(\mathrm{A}_{2}\right)$ that

$$
\begin{aligned}
& 0 \leq \tilde{q}^{*}(t) \Gamma S_{1} \Gamma \tilde{q}(t)-f^{*}(\tilde{q}(t)) S_{1} f(\tilde{q}(t)), \\
& 0 \leq \tilde{q}^{*}(t-\tau) \Gamma S_{2} \Gamma \tilde{q}(t-\tau)-f^{*}(\tilde{q}(t-\tau)) S_{2} f(\tilde{q}(t-\tau)) .
\end{aligned}
$$

From system (12), it is obvious that

$$
\begin{aligned}
0= & {\left[Q_{1} \dot{\tilde{q}}(t)+Q_{2} \tilde{q}(t-\delta)\right]^{*}[-\dot{\tilde{q}}(t)-D \tilde{q}(t-\delta)+A f(\tilde{q}(t))} \\
& \left.+B f(\tilde{q}(t-\tau))+C \int_{-\infty}^{t} k(t-s) f(q(s)) \mathrm{d} s\right] \\
& +[-\dot{\tilde{q}}(t)-D \tilde{q}(t-\delta)+A f(\tilde{q}(t))+B f(\tilde{q}(t-\tau)) \\
& \left.+C \int_{-\infty}^{t} k(t-s) f(q(s)) \mathrm{d} s\right]^{*}\left[Q_{1} \dot{\tilde{q}}(t)+Q_{2} \tilde{q}(t-\delta)\right] .
\end{aligned}
$$


It follows from equalities or inequalities (18)-(25) and Lemma 4 that

$$
\begin{aligned}
& \dot{V}(t) \leq \tilde{q}^{*}(t)\left(-P_{1} D-D P_{1}+P_{2}+\delta^{2} P_{3}+P_{4}+\Gamma R \Gamma+\Gamma S_{1} \Gamma\right) \tilde{q}(t) \\
& +\tilde{q}^{*}(t) P_{1} A f(\tilde{q}(t))+f^{*}(\tilde{q}(t)) A^{*} P_{1} \tilde{q}(t)+\tilde{q}^{*}(t) P_{1} B f(\tilde{q}(t-\tau)) \\
& +f^{*}(\tilde{q}(t-\tau)) B^{*} P_{1} \tilde{q}(t)+\tilde{q}^{*}(t) P_{1} C\left[\int_{-\infty}^{t} k(t-s) f(q(s)) \mathrm{d} s\right] \\
& +\left[\int_{-\infty}^{t} k(t-s) f(q(s)) \mathrm{d} s\right]^{*} C^{*} P_{1} \tilde{q}(t)+\tilde{q}^{*}(t) D P_{1} D\left[\int_{t-\delta}^{t} \tilde{q}(s) \mathrm{d} s\right] \\
& +\left[\int_{t-\delta}^{t} \tilde{q}(s) \mathrm{d} s\right]^{*} D P_{1} D \tilde{q}(t)+\dot{\tilde{q}}^{*}(t)\left(-Q_{1}^{*}-Q_{1}\right) \dot{\tilde{q}}(t) \\
& +\dot{\tilde{q}}^{*}(t)\left(-Q_{1}^{*} D-Q_{2}\right) \tilde{q}(t-\delta)+\tilde{q}^{*}(t-\delta)\left(-D Q_{1}-Q_{2}^{*}\right) \dot{\tilde{q}}(t) \\
& +\dot{\tilde{q}}^{*}(t) Q_{1}^{*} A f(\tilde{q}(t))+f^{*}(\tilde{q}(t)) A^{*} Q_{1} \dot{\tilde{q}}(t)+\dot{\tilde{q}}^{*}(t) Q_{1}^{*} B f(\tilde{q}(t-\tau)) \\
& +f^{*}(\tilde{q}(t-\tau)) B^{*} Q_{1} \dot{\tilde{q}}(t)+\dot{\tilde{q}}^{*}(t) Q_{1}^{*} C\left[\int_{-\infty}^{t} k(t-s) f(q(s)) \mathrm{d} s\right] \\
& +\left[\int_{-\infty}^{t} k(t-s) f(q(s)) \mathrm{d} s\right]^{*} C^{*} Q_{1} \dot{\tilde{q}}(t) \\
& +\tilde{q}^{*}(t-\delta)\left(-P_{2}-Q_{2}^{*} D-D Q_{2}\right) \tilde{q}(t-\delta) \\
& +\tilde{q}^{*}(t-\delta) Q_{2}^{*} A f(\tilde{q}(t))+f^{*}(\tilde{q}(t)) A^{*} Q_{2} \tilde{q}(t-\delta) \\
& +\tilde{q}^{*}(t-\delta) Q_{2}^{*} B f(\tilde{q}(t-\tau))+f^{*}(\tilde{q}(t-\tau)) B^{*} Q_{2} \tilde{q}(t-\delta) \\
& +\tilde{q}^{*}(t-\delta) Q_{2}^{*} C\left[\int_{-\infty}^{t} k(t-s) f(q(s)) \mathrm{d} s\right] \\
& +\left[\int_{-\infty}^{t} k(t-s) f(q(s)) \mathrm{d} s\right]^{*} C Q_{2} \tilde{q}(t-\delta) \\
& +\tilde{q}^{*}(t-\tau)\left(-P_{4}+\Gamma S_{2} \Gamma\right) \tilde{q}(t-\tau)-f^{*}(\tilde{q}(t)) S_{1} f(\tilde{q}(t)) \\
& -f^{*}(\tilde{q}(t)) A^{*} P_{1} D\left[\int_{t-\delta}^{t} \tilde{q}(s) \mathrm{d} s\right]-\left[\int_{t-\delta}^{t} \tilde{q}(s) \mathrm{d} s\right]^{*} D P_{1} A f(\tilde{q}(t)) \\
& -f^{*}(\tilde{q}(t-\tau)) S_{2} f(\tilde{q}(t-\tau))-f^{*}(\tilde{q}(t-\tau)) B^{*} P_{1} D\left[\int_{t-\delta}^{t} \tilde{q}(s) \mathrm{d} s\right] \\
& -\left[\int_{t-\delta}^{t} \tilde{q}(s) \mathrm{d} s\right]^{*} D P_{1} B f(\tilde{q}(t-\tau))-\left[\int_{-\infty}^{t} k(t-s) f(q(s)) \mathrm{d} s\right]^{*} R \\
& \times\left[\int_{-\infty}^{t} k(t-s) f(q(s)) \mathrm{d} s\right]-\left[\int_{t-\delta}^{t} \tilde{q}(s) \mathrm{d} s\right]^{*} P_{3}\left[\int_{t-\delta}^{t} \tilde{q}(s) \mathrm{d} s\right] \\
& -\left[\int_{-\infty}^{t} k(t-s) f(q(s)) \mathrm{d} s\right]^{*} C^{*} P_{1} D\left[\int_{t-\delta}^{t} \tilde{q}(s) \mathrm{d} s\right] \\
& -\left[\int_{t-\delta}^{t} \tilde{q}(s) \mathrm{d} s\right]^{*} D P_{1} C\left[\int_{-\infty}^{t} k(t-s) f(q(s)) \mathrm{d} s\right] \\
& \leq|\tilde{q}(t)|^{T}\left(-P_{1} \check{D}-\check{D} P_{1}+P_{2}+\delta^{2} P_{3}+P_{4}+\Gamma R \Gamma+\Gamma S_{1} \Gamma\right)|\tilde{q}(t)| \\
& +|\tilde{q}(t)|^{T} P_{1} \tilde{A}|f(\tilde{q}(t))|+|f(\tilde{q}(t))|^{T} \tilde{A}^{T} P_{1}|\tilde{q}(t)|+|\tilde{q}(t)|^{T} P_{1} \tilde{B}|f(\tilde{q}(t-\tau))| \\
& +|f(\tilde{q}(t-\tau))|^{T} \tilde{B}^{T} P_{1}|\tilde{q}(t)|+|\tilde{q}(t)|^{T} P_{1} \tilde{C}\left|\int_{-\infty}^{t} k(t-s) f(q(s)) \mathrm{d} s\right|
\end{aligned}
$$




$$
\begin{aligned}
& +\left|\int_{-\infty}^{t} k(t-s) f(q(s)) \mathrm{d} s\right|^{T} \tilde{C}^{T} P_{1}|\tilde{q}(t)|+|\tilde{q}(t)|^{T} \hat{D} P_{1} \hat{D}\left|\int_{t-\delta}^{t} \tilde{q}(s) \mathrm{d} s\right| \\
& +\left|\int_{t-\delta}^{t} \tilde{q}(s) \mathrm{d} s\right|^{T} \hat{D} P_{1} \hat{D}|\tilde{q}(t)|+|\dot{\tilde{q}}(t)|^{T}\left(-Q_{1}^{T}-Q_{1}\right)|\dot{\tilde{q}}(t)| \\
& +|\dot{\tilde{q}}(t)|^{T}\left(Q_{1}^{T} \hat{D}+Q_{2}\right)|\tilde{q}(t-\delta)|+|\tilde{q}(t-\delta)|^{T}\left(\hat{D} Q_{1}+Q_{2}^{T}\right)|\dot{\tilde{q}}(t)| \\
& +|\dot{\tilde{q}}(t)|^{T} Q_{1}^{T} \tilde{A}|f(\tilde{q}(t))|+|f(\tilde{q}(t))|^{T} \tilde{A}^{T} Q_{1}|\dot{\tilde{q}}(t)|+|\dot{\tilde{q}}(t)|^{T} Q_{1}^{T} \tilde{B}|f(\tilde{q}(t-\tau))| \\
& +|f(\tilde{q}(t-\tau))|^{T} \tilde{B}^{T} Q_{1}|\dot{\tilde{q}}(t)|+|\dot{\tilde{q}}(t)|^{T} Q_{1}^{T} \tilde{C}\left|\int_{-\infty}^{t} k(t-s) f(q(s)) \mathrm{d} s\right| \\
& +\left|\int_{-\infty}^{t} k(t-s) f(q(s)) \mathrm{d} s\right|^{T} \tilde{C}^{T} Q_{1}|\dot{\tilde{q}}(t)| \\
& +|\tilde{q}(t-\delta)|^{T}\left(-P_{2}-Q_{2}^{T} \check{D}-\check{D} Q_{2}\right)|\tilde{q}(t-\delta)| \\
& +|\tilde{q}(t-\delta)|^{T} Q_{2}^{T} \tilde{A}|f(\tilde{q}(t))|+|f(\tilde{q}(t))|^{T} \tilde{A}^{T} Q_{2}|\tilde{q}(t-\delta)| \\
& +|\tilde{q}(t-\delta)|^{T} Q_{2}^{T} \tilde{B}|f(\tilde{q}(t-\tau))|+|f(\tilde{q}(t-\tau))|^{T} \tilde{B}^{T} Q_{2}|\tilde{q}(t-\delta)| \\
& +|\tilde{q}(t-\delta)|^{T} Q_{2}^{T} \tilde{C}\left|\int_{-\infty}^{t} k(t-s) f(q(s)) \mathrm{d} s\right| \\
& +\left|\int_{-\infty}^{t} k(t-s) f(q(s)) \mathrm{d} s\right|^{T} \tilde{C} Q_{2}|\tilde{q}(t-\delta)| \\
& +|\tilde{q}(t-\tau)|^{T}\left(-P_{4}+\Gamma S_{2} \Gamma\right)|\tilde{q}(t-\tau)|-|f(\tilde{q}(t))|^{T} S_{1}|f(\tilde{q}(t))| \\
& +|f(\tilde{q}(t))|^{T} \tilde{A}^{T} P_{1} \hat{D}\left|\int_{t-\delta}^{t} \tilde{q}(s) \mathrm{d} s\right|+\left|\int_{t-\delta}^{t} \tilde{q}(s) \mathrm{d} s\right|^{T} \hat{D} P_{1} \tilde{A}|f(\tilde{q}(t))| \\
& -|f(\tilde{q}(t-\tau))|^{T} S_{2}|f(\tilde{q}(t-\tau))|+|f(\tilde{q}(t-\tau))|^{T} \tilde{B}^{T} P_{1} \hat{D}\left|\int_{t-\delta}^{t} \tilde{q}(s) \mathrm{d} s\right| \\
& +\left|\int_{t-\delta}^{t} \tilde{q}(s) \mathrm{d} s\right|^{T} \hat{D} P_{1} \tilde{B}|f(\tilde{q}(t-\tau))|-\left|\int_{-\infty}^{t} k(t-s) f(q(s)) \mathrm{d} s\right|^{T} R \\
& \times\left|\int_{-\infty}^{t} k(t-s) f(q(s)) \mathrm{d} s\right|-\left|\int_{t-\delta}^{t} \tilde{q}(s) \mathrm{d} s\right|^{T} P_{3}\left|\int_{t-\delta}^{t} \tilde{q}(s) \mathrm{d} s\right| \\
& +\left|\int_{-\infty}^{t} k(t-s) f(q(s)) \mathrm{d} s\right|^{T} \tilde{C}^{T} P_{1} \hat{D}\left|\int_{t-\delta}^{t} \tilde{q}(s) \mathrm{d} s\right| \\
& +\left|\int_{t-\delta}^{t} \tilde{q}(s) \mathrm{d} s\right|^{T} \hat{D} P_{1} \tilde{C}\left|\int_{-\infty}^{t} k(t-s) f(q(s)) \mathrm{d} s\right| \\
& \leq \alpha^{T} \Omega \alpha
\end{aligned}
$$

where

$$
\begin{aligned}
\alpha= & \left(\left|\tilde{q}^{T}(t)\right|,\left|\dot{\tilde{q}}^{T}(t)\right|,\left|\tilde{q}^{T}(t-\delta)\right|,\left|\tilde{q}^{T}(t-\tau)\right|,\left|f^{T}(\tilde{q}(t))\right|,\right. \\
& \left.\left|f^{T}(\tilde{q}(t-\tau))\right|,\left|\int_{-\infty}^{t} k(t-s) f(q(s)) \mathrm{d} s\right|^{T},\left|\int_{t-\delta}^{t} \tilde{q}(s) \mathrm{d} s\right|^{T}\right)^{T} .
\end{aligned}
$$

Therefore, we conclude that $\dot{V}(t)$ is negative definite because of LMI (9). Moreover, it is obvious that $V(t)$ is radially unbounded. Then the equilibrium point of system (1) is globally asymptotically stable by standard Lyapunov theorem. 
Remark 2 It should be noted that RVNNs and CVNNs are special cases of QVNNs. So the results of the paper can also be applied to RVNNs and CVNNs in the form of (1).

\section{Numerical examples}

In this section, two numerical examples will illustrate the effectiveness of the proposed results.

Example 1 Suppose that the parameters of QVNNs (1) are given as follows:

$$
\begin{aligned}
& \check{D}=\left(\begin{array}{cc}
0.3 & 0 \\
0 & 0.3
\end{array}\right), \quad \hat{D}=\left(\begin{array}{cc}
0.32 & 0 \\
0 & 0.32
\end{array}\right), \\
& \check{A}=\left(\check{a}_{i j}\right)_{2 \times 2}, \quad \hat{A}=\left(\hat{a}_{i j}\right)_{2 \times 2}, \\
& \check{B}=\left(\check{b}_{i j}\right)_{2 \times 2}, \quad \hat{B}=\left(\hat{b}_{i j}\right)_{2 \times 2}, \\
& \check{C}=\left(\check{c}_{i j}\right)_{2 \times 2}, \quad \hat{C}=\left(\hat{c}_{i j}\right)_{2 \times 2}, \\
& \Gamma=\left(\begin{array}{cc}
0.2 & 0 \\
0 & 0.2
\end{array}\right), \quad \delta=0.5, \quad \tau=1,
\end{aligned}
$$

where

$$
\begin{aligned}
& \check{a}_{11}=-0.1-0.1 \imath-0.1 J-0.1 \kappa, \\
& \check{a}_{12}=-0.1-0.1 \imath-0.1 \jmath-0.1 \kappa, \\
& \check{a}_{21}=-0.15-0.15 \imath-0.15 \jmath-0.15 \kappa, \\
& \check{a}_{22}=-0.1-0.1 \imath-0.1 \jmath-0.1 \kappa, \\
& \hat{a}_{11}=0.1+0.1 \imath+0.1 J+0.1 \kappa, \\
& \hat{a}_{12}=0.1+0.1 \imath+0.1 J+0.1 \kappa, \\
& \hat{a}_{21}=0.15+0.15 \imath+0.15 J+0.15 \kappa, \\
& \hat{a}_{22}=0.1+0.1 \imath+0.1 J+0.1 \kappa, \\
& \check{b}_{11}=-0.1-0.1 \imath-0.1 J-0.1 \kappa, \\
& \check{b}_{12}=-0.05-0.05 \imath-0.05 J-0.05 \kappa, \\
& \check{b}_{21}=-0.1-0.1 \imath-0.1 J-0.1 \kappa, \\
& \check{b}_{22}=-0.1-0.1 \imath-0.1 J-0.1 \kappa, \\
& \hat{b}_{11}=0.1+0.1 \imath+0.1 J+0.1 \kappa, \\
& \hat{b}_{12}=0.05+0.05 \imath+0.05 J+0.05 \kappa, \\
& \hat{b}_{21}=0.1+0.1 \imath+0.1 J+0.1 \kappa, \\
& \hat{b}_{22}=0.1+0.1 \imath+0.1 J+0.1 \kappa, \\
& \check{c}_{11}=-0.05-0.05 \imath-0.05 J-0.05 \kappa, \\
& \check{c}_{12}=-0.075-0.075 \imath-0.075 J-0.075 \kappa,
\end{aligned}
$$




$$
\begin{aligned}
& \check{c}_{21}=-0.1-0.1 \imath-0.1 \jmath-0.1 \kappa, \\
& \check{c}_{22}=-0.05-0.05 \imath-0.05 \jmath-0.05 \kappa, \\
& \hat{c}_{11}=0.05+0.05 \imath+0.05 \jmath+0.05 \kappa, \\
& \hat{c}_{12}=0.075+0.075 \imath+0.075 \jmath+0.075 \kappa, \\
& \hat{c}_{21}=0.1+0.1 \imath+0.1 \jmath+0.1 \kappa, \\
& \hat{c}_{22}=0.05+0.05 \imath+0.05 \jmath+0.05 \kappa .
\end{aligned}
$$

According to the matrices $\check{A}, \hat{A}, \check{B}, \hat{B}, \check{C}$ and $\hat{C}$, we get the following matrices:

$$
\tilde{A}=\left(\begin{array}{ll}
0.2 & 0.2 \\
0.3 & 0.2
\end{array}\right), \quad \tilde{B}=\left(\begin{array}{cc}
0.2 & 0.1 \\
0.2 & 0.2
\end{array}\right), \quad \tilde{C}=\left(\begin{array}{cc}
0.1 & 0.15 \\
0.2 & 0.1
\end{array}\right)
$$

Then, by using YALMIP with solver of SDPT3 in MATLAB, we obtain the feasible solutions of LMI (9) in Theorem 2 as follows:

$$
\begin{array}{ll}
P_{1}=\left(\begin{array}{cc}
36.4456 & 0 \\
0 & 23.2727
\end{array}\right), & P_{2}=\left(\begin{array}{cc}
0.0807 & 0 \\
0 & 0.0625
\end{array}\right), \\
P_{3}=\left(\begin{array}{cc}
12.6787 & 0 \\
0 & 7.8742
\end{array}\right), & P_{4}=\left(\begin{array}{cc}
3.0234 & 0 \\
0 & 1.7847
\end{array}\right), \\
Q_{1}=\left(\begin{array}{cc}
0.3209 & 0 \\
0 & 0.2297
\end{array}\right), & Q_{2}=\left(\begin{array}{cc}
0.0793 & 0 \\
0 & 0.0647
\end{array}\right), \\
R=\left(\begin{array}{cc}
54.7847 & 0 \\
0 & 38.6931
\end{array}\right), & S_{1}=\left(\begin{array}{cc}
91.8249 & 0 \\
0 & 60.4696
\end{array}\right), \\
S_{2}=\left(\begin{array}{cc}
75.0553 & 0 \\
0 & 43.9460
\end{array}\right) . &
\end{array}
$$

Therefore, according to Theorem 2, the QVNNs (1) have a unique equilibrium point which is globally robust stable.

In what follows, we consider a special model in this example and give simulation results for the sake of verification of the proposed results. We choose the following fixed network parameters:

$$
\begin{aligned}
& D=\left(\begin{array}{cc}
0.3 & 0 \\
0 & 0.32
\end{array}\right), \quad A=\left(a_{i j}\right)_{2 \times 2}, \quad B=\left(b_{i j}\right)_{2 \times 2}, \\
& C=\left(c_{i j}\right)_{2 \times 2}, \quad J=\left(\begin{array}{c}
0.1-0.1 l-0.2 J+0.05 \kappa \\
-0.2+0.1 l+0.05 J-0.1 \kappa
\end{array}\right),
\end{aligned}
$$

where

$$
\begin{aligned}
& a_{11}=0.1-0.1 \imath+0.08 J-0.1 \kappa, \\
& a_{12}=-0.1+0.1 \imath-0.1 \jmath+0.05 \kappa,
\end{aligned}
$$




$$
\begin{aligned}
& a_{21}=0.15+0.1 \imath-0.15 \jmath-0.1 \kappa, \\
& a_{22}=0.08-0.08 \imath+0.1 \jmath+0.1 \kappa, \\
& b_{11}=0.1-0.06 \imath+0.1 \jmath+0.09 \kappa, \\
& b_{12}=0.05+0.05 \imath-0.05 \jmath-0.03 \kappa, \\
& b_{21}=-0.1+0.1 \imath-0.08 \jmath-0.05 \kappa, \\
& b_{22}=0.1-0.1 \imath+0.1 \jmath+0.08 \kappa, \\
& c_{11}=0.05-0.03 \imath+0.05 \jmath-0.04 \kappa, \\
& c_{12}=-0.07+0.05 \imath-0.06 \jmath+0.05 \kappa, \\
& c_{21}=0.1+0.1 \imath-0.1 \jmath-0.08 \kappa, \\
& c_{22}=0.05-0.05 \imath+0.05 \jmath+0.03 \kappa .
\end{aligned}
$$

Besides, we choose the following functions as the activations and the delay kernel:

$$
\begin{aligned}
& f_{1}(u)=f_{2}(u)=0.1(|u+1|-|u-1|), \quad \forall u \in \mathbb{H}, \\
& k(s)=e^{-s}, \quad \forall s \in[0,+\infty) .
\end{aligned}
$$

Based on these fixed parameters, we perform numerical simulation of the system by employing the fourth-order Runge-Kutta methods. Figures 1, 2, 3 and 4 show that the four parts of the states of the considered system, where the initial conditions are chosen by 10 random constant vectors. It can be seen from these figures that each neuron state converges to the stable equilibrium point, which is $(0.4339-0.4488 l-0.5384 J+$ $0.1301 \kappa,-0.6699+0.4545 \imath+0.0010 \jmath-0.4327 \kappa)^{T}$.

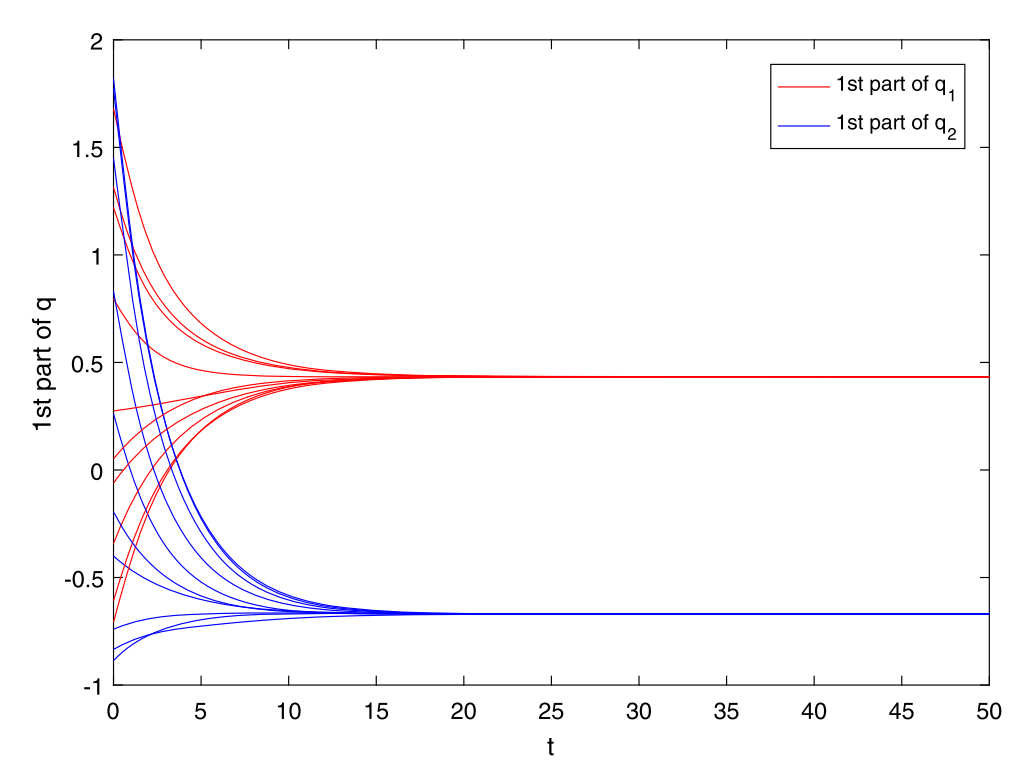

Figure 1 The first part of the state trajectories for system (1) with parameters (27) 


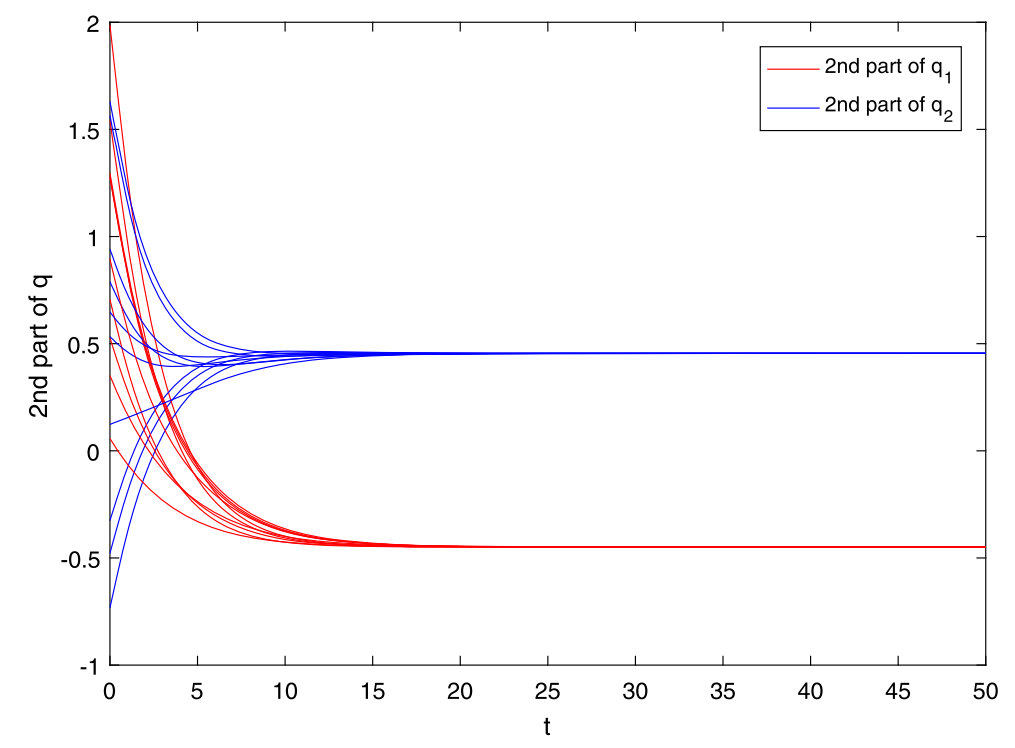

Figure 2 The second part of the state trajectories for system (1) with parameters (27)

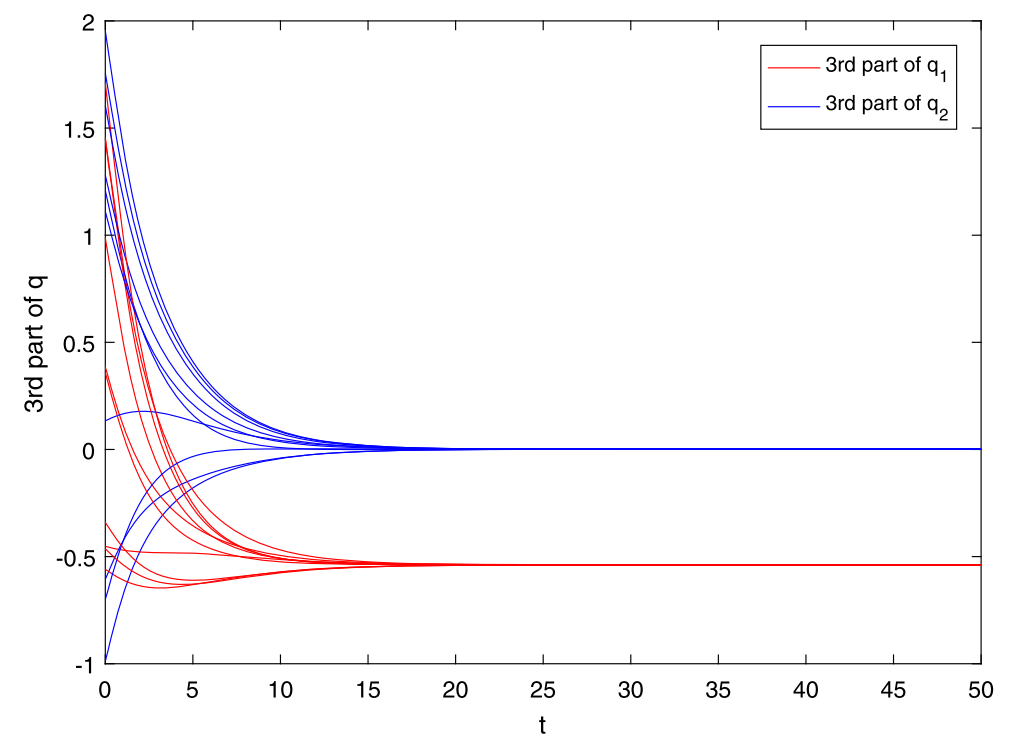

Figure 3 The third part of the state trajectories for system (1) with parameters (27)

Remark 3 Although the NNs (1) are quaternion-valued, the stability criteria are expressed in the form of LMIs (4) and (9), which are real-valued. In Example 1, we see that these LMIs can be checked directly by the mathematical software MATLAB.

Remark 4 In [35], the authors considered the robust stability of QVNNs with both leakage and discrete delays but without distributed delay. The criteria obtained in [35] cannot be applied to check the robust stability of the system in Example 1, since the system has distributed delay. 


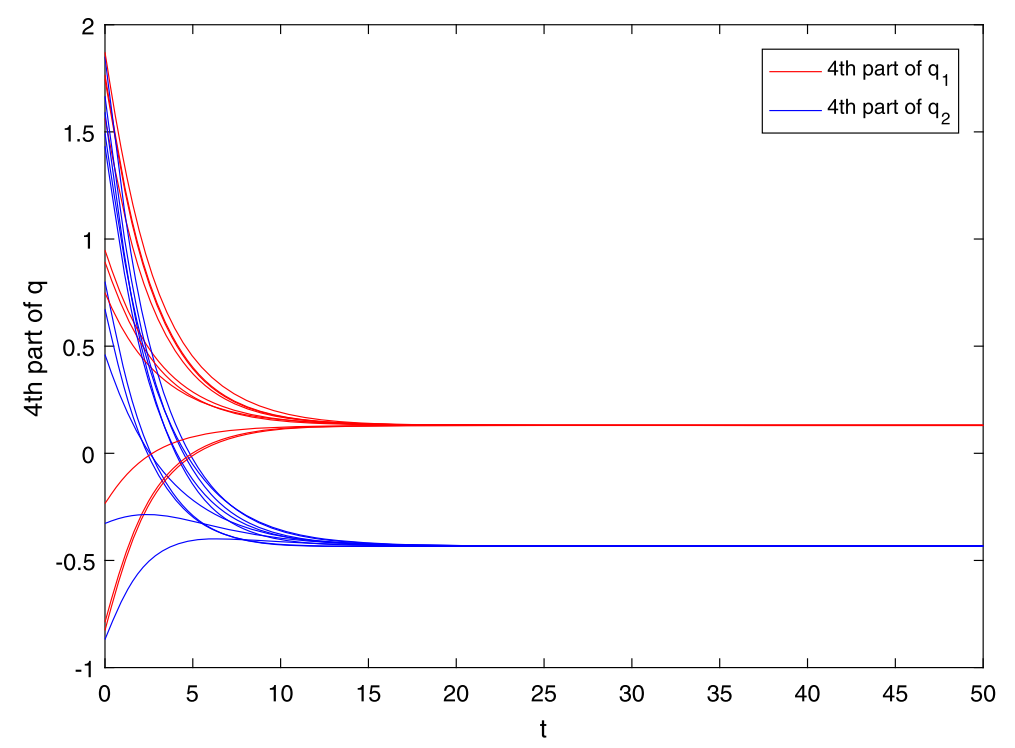

Figure 4 The fourth part of the state trajectories for system (1) with parameters (27)

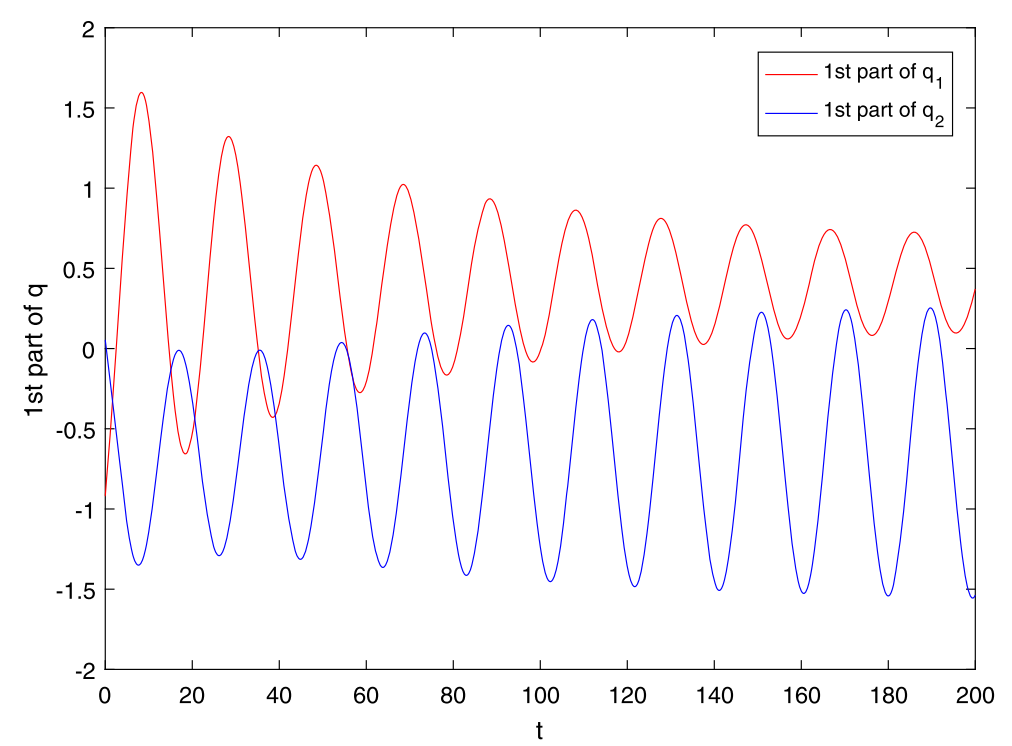

Figure 5 The first part of the state trajectories for system (1) with $\delta=4.6$

Remark 5 For investigating the effects of time delays on the system, we set leakage delay $\delta=4.6,5.5$, and 8 in Example 1. Then we find that the equilibrium point of the system is unstable. Figures 5, 6 and 7 show that the first part of the states of the system do not converge to the equilibrium point.

Example 2 Suppose that the parameters of system (1) are given as follows:

$$
\check{D}=\left(\begin{array}{cc}
0.3 & 0 \\
0 & 0.5
\end{array}\right), \quad \hat{D}=\left(\begin{array}{cc}
0.5 & 0 \\
0 & 0.7
\end{array}\right) \text {, }
$$




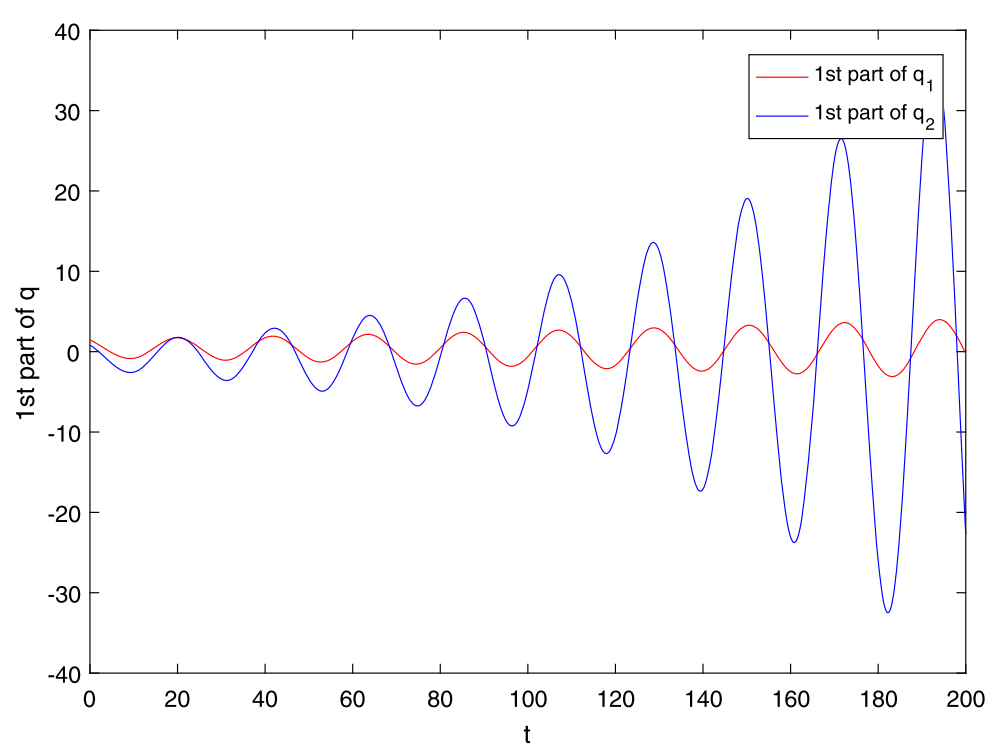

Figure 6 The first part of the state trajectories for system (1) with $\delta=5.5$

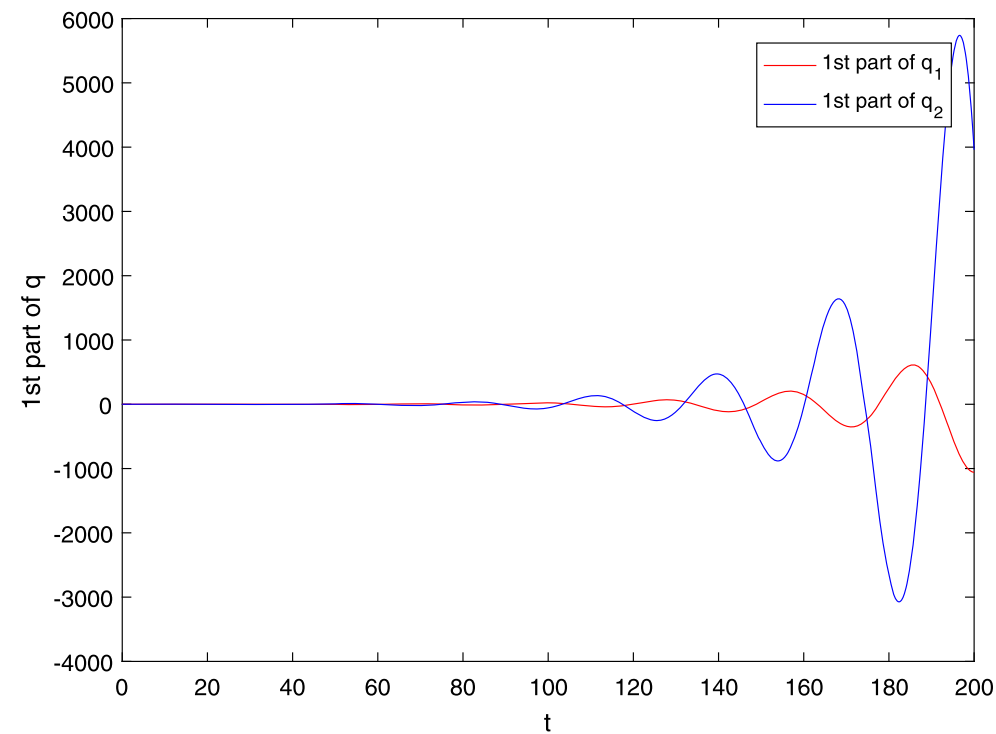

Figure 7 The first part of the state trajectories for system (1) with $\delta=8$

$$
\begin{aligned}
& \check{A}=\left(\begin{array}{ll}
-0.4-0.3 l & -0.32-0.24 \imath \\
-0.3-0.4 \imath & -0.54-0.72 \imath
\end{array}\right), \quad \hat{A}=\left(\begin{array}{cc}
0.3+0.4 l & 0.32+0.24 \imath \\
0.3+0.4 l & 0.72+0.54 \imath
\end{array}\right), \\
& \check{B}=\left(\begin{array}{cc}
-0.24-0.32 \imath & -0.18-0.24 \iota \\
-0.3-0.4 \imath & -0.32-0.24 \iota
\end{array}\right), \quad \hat{B}=\left(\begin{array}{cc}
0.32+0.24 \imath & 0.18+0.24 \imath \\
0.4+0.3 \imath & 0.24+0.32 \iota
\end{array}\right) \text {, } \\
& \check{C}=\hat{C}=\left(\begin{array}{ll}
0 & 0 \\
0 & 0
\end{array}\right), \quad \Gamma=\left(\begin{array}{cc}
0.2 & 0 \\
0 & 0.2
\end{array}\right), \quad \delta=0, \quad \tau=2 .
\end{aligned}
$$


According to the matrices $\check{A}, \hat{A}, \check{B}, \hat{B}, \check{C}$ and $\hat{C}$, we get the following matrices:

$$
\tilde{A}=\left(\begin{array}{ll}
0.5 & 0.4 \\
0.5 & 0.9
\end{array}\right), \quad \tilde{B}=\left(\begin{array}{ll}
0.4 & 0.3 \\
0.5 & 0.4
\end{array}\right), \quad \tilde{C}=\left(\begin{array}{ll}
0 & 0 \\
0 & 0
\end{array}\right) .
$$

Then, by using YALMIP with solver of SDPT3 in MATLAB, we obtain the feasible solutions of LMI (9) in Theorem 2 as follows:

$$
\begin{aligned}
& P_{1}=\left(\begin{array}{cc}
10.0939 & 0 \\
0 & 7.0398
\end{array}\right), \quad P_{2}=\left(\begin{array}{cc}
0.0058 & 0 \\
0 & 0.0077
\end{array}\right), \\
& P_{3}=\left(\begin{array}{cc}
974.6 & 0 \\
0 & 1190.1
\end{array}\right), \quad P_{4}=\left(\begin{array}{cc}
1.4122 & 0 \\
0 & 1.2880
\end{array}\right), \\
& Q_{1}=\left(\begin{array}{cc}
0.0120 & 0 \\
0 & 0.0075
\end{array}\right), \quad Q_{2}=\left(\begin{array}{cc}
0.0053 & 0 \\
0 & 0.0040
\end{array}\right), \\
& R=\left(\begin{array}{cc}
0.0609 & 0 \\
0 & 0.0848
\end{array}\right), \quad S_{1}=\left(\begin{array}{cc}
40.2801 & 0 \\
0 & 55.7370
\end{array}\right), \\
& S_{2}=\left(\begin{array}{cc}
35.2199 & 0 \\
0 & 32.0899
\end{array}\right) .
\end{aligned}
$$

Therefore, according to Theorem 2, system (1) has a unique equilibrium point which is globally robust stable.

Remark 6 In Example 2, the system parameters are complex-valued. So the system with these parameters can be viewed as CVNNs. Moreover, since $\delta=0$ and $\check{C}=\hat{C}=0$, the CVNNs have no leakage delay nor distributed delay. Then we try to apply the criteria in [22] to check the robust stability of the CVNNs. Via YALMIP with solver of SDPT3 in MATLAB, we cannot find feasible solutions of LMIs in [22]. Therefore, the results obtained in [22] cannot be applied to check the robust stability of the CVNNs.

\section{Conclusion}

In this paper, the robust stability problem of parametric uncertain QVNNs with both leakage, discrete and distributed delays has been investigated. Based on Homeomorphic mapping theorem and Lyapunov theorem, some criteria are obtained to check the existence, uniqueness, and global robust stability of the equilibrium point of the delayed QVNNs. Owing to using LMI approach and the modulus inequality technique, the presentation of the obtained criteria is in the form of real-valued LMIs, which can be solved by the mathematical software MATLAB directly and feasibly. In addition, two numerical examples are provided to substantiate the effectiveness of the proposed LMI conditions. It should be noted that the activation functions are continuous in this paper. Considering that the discontinuous neural network is one of the important dynamic systems, therefore, our further works will research the stability problem of QVNNs with discontinuous activations. 


\section{Competing interests}

The authors declare that they have no competing interests.

\section{Authors' contributions}

XC conceived, designed and performed the experiments. XC, LL and ZL wrote the paper. All authors read and approved the final manuscript.

\section{Author details}

${ }^{1}$ Department of Mathematics, Chongqing Jiaotong University, Chongqing, China. ${ }^{2}$ Department of Mathematics and Statistics, Georgia State University, Atlanta, USA.

\section{Publisher's Note}

Springer Nature remains neutral with regard to jurisdictional claims in published maps and institutional affiliations.

Received: 14 December 2017 Accepted: 3 April 2018 Published online: 12 April 2018

\section{References}

1. Simmons, G.F:: Calculus Gems: Brief Lives and Memorable Mathematics. McGraw-Hill, New York (1992)

2. Conway, J.H., Smith, D.A.: On Quaternions and Octonions: Their Geometry, Arithmetic, and Symmetry. AK Peters, Natick (2003)

3. Matsui, N., Isokawa, T., Kusamichi, H., Peper, F., Nishimura, H.: Quaternion neural network with geometrical operators. J. Intell. Fuzzy Syst. 15(3-4), 149-164 (2004)

4. Adler, S.L.: Quaternionic Quantum Mechanics and Quantum Fields. Oxford University Press, New York (1995)

5. Ujang, B.C., Took, C.C., Mandic, D.P.: Quaternion-valued nonlinear adaptive filtering. IEEE Trans. Neural Netw. 22(8), 1193-1206 (2011)

6. Mazinan, A.H., Pasand, M., Soltani, B.: Full quaternion based finite-time cascade attitude control approach via pulse modulation synthesis for a spacecraft. ISA Trans. 58, 567-585 (2015)

7. Zeng, Z., Wang, J.: Design and analysis of high-capacity associative memories based on a class of discrete-time recurrent neural networks. IEEE Trans. Syst. Man Cybern., Part B, Cybern. 38(6), 1525-1536 (2008)

8. Lu, J., Ho, D.W.C., Wu, L.: Exponential stabilization of switched stochastic dynamical networks. Nonlinearity 22(4), 889-911 (2009)

9. Tanaka, G., Aihara, K.: Complex-valued multistate associative memory with nonlinear multilevel functions for gray-level image reconstruction. IEEE Trans. Neural Netw. 20, 1463-1473 (2009)

10. Lu, J., Ho, D.W.C.: Stabilization of complex dynamical networks with noise disturbance under performance constraint. Nonlinear Anal., Real World Appl. 12(4), 1974-1984 (2011)

11. Zhang, W., Tang, Y., Miao, Q., Du, W.: Exponential synchronization of coupled switched neural networks with mode-dependent impulsive effects. IEEE Trans. Neural Netw. Learn. Syst. 24(8), 1316-1326 (2013)

12. Zhang, W., Tang, Y., Wu, X., Fang, J.A.: Synchronization of nonlinear dynamical networks with heterogeneous impulses. IEEE Trans. Circuits Syst. I, Regul. Pap. 61(4), 1220-1228 (2014)

13. Yang, R., Wu, B., Liu, Y.: A Halanay-type inequality approach to the stability analysis of discrete-time neural networks with delays. Appl. Math. Comput. 265, 696-707 (2015)

14. Wang, J.L., Wu, H.N., Huang, T., Ren, S.Y., Wu, J.: Pinning control for synchronization of coupled reaction-diffusion neural networks with directed topologies. IEEE Trans. Syst. Man Cybern. Syst. 46(8), 1109-1120 (2016)

15. Sun, C., He, W., Ge, W., Chang, C.: Adaptive neural network control of biped robots. IEEE Trans. Syst. Man Cybern. Syst. 47(2), 315-326 (2017)

16. Zhang, W., Tang, Y., Huang, T., Kurths, J.: Sampled-data consensus of linear multi-agent systems with packet losses. IEEE Trans. Neural Netw. Learn. Syst. 28(11), 2516-2527 (2017)

17. Rakkiyappan, R., Udhayakumar, K., Velmurugan, G., Cao, J., Alsaedi, A.: Stability and Hopf bifurcation analysis of fractional-order complex-valued neural networks with time delays. Adv. Differ. Equ. 2017(1), 225 (2017)

18. Zhang, X., Li, C., Huang, T.: Impacts of state-dependent impulses on the stability of switching Cohen-Grossberg neural networks. Adv. Differ. Equ. 2017(1), 316 (2017)

19. Chen, X., Zhao, Z., Song, Q., Hu, J.: Multistability of complex-valued neural networks with time-varying delays. Appl. Math. Comput. 294, 18-35 (2017)

20. Shen, H., Zhu, Y., Zhang, L., Park, J.H.: Extended dissipative state estimation for Markov jump neural networks with unreliable links. IEEE Trans. Neural Netw. Learn. Syst. 28(2), 346-358 (2017)

21. Shi, Y., Cao, J., Chen, G.: Exponential stability of complex-valued memristor-based neural networks with time-varying delays. Appl. Math. Comput. 313, 222-234 (2017)

22. Tan, Y., Tang, S., Yang, J., Liu, Z.: Robust stability analysis of impulsive complex-valued neural networks with time delays and parameter uncertainties. J. Inequal. Appl. 2017, 215 (2017)

23. Liu, Y., Xu, P., Lu, J., Liang, J.: Global stability of Clifford-valued recurrent neural networks with time delays. Nonlinear Dyn. 84(2), 767-777 (2016)

24. Zhang, D., Kou, K.I., Liu, Y., Cao, J.: Decomposition approach to the stability of recurrent neural networks with asynchronous time delays in quaternion field. Neural Netw. 94, 55-66 (2017)

25. Isokawa, T., Kusakabe, T., Matsui, N., Peper, F.: Quaternion neural network and its application. In: Proc. 7th Int. Conf. KES, Oxford, UK, pp. 318-324 (2003)

26. Luo, L., Feng, H., Ding, L.: Color image compression based on quaternion neural network principal component analysis. In: Proc. Int. Conf. Multimedia Technol., pp. 1-4 (2010)

27. Kusamichi, H., Isokawa, T., Matsui, N., Ogawa, Y., Maeda, K.: A new scheme for color night vision by quaternion neural network. In: Proc. 2nd Int. Conf. Auton. Robots Agents, pp. 101-106 (2004)

28. Isokawa, T., Nishimura, H., Kamiura, N., Matsui, N.: Associative memory in quaternionic Hopfield neural network. Int. J. Neural Syst. 18(2), 135-145 (2008) 
29. Minemoto, T., Isokawa, T., Nishimura, H., Matsui, N.: Quaternionic multistate Hopfield neural network with extended projection rule. Artif. Life Robot. 21(1), 106-111 (2016)

30. Chen, X., Song, Q., Li, Z.: Design and analysis of quaternion-valued neural networks for associative memories. IEEE Trans. Syst. Man Cybern. Syst. (2017). http://ieeexplore.ieee.org/document/7970154/. https://doi.org/10.1109/TSMC.2017.2717866

31. Liu, Y., Zhang, D., Lou, J., Lu, J., Cao, J.: Stability analysis of quaternion-valued neural networks: decomposition and direct approaches. IEEE Trans. Neural Netw. Learn. Syst. (2017). http://ieeexplore.ieee.org/document/8088357/. https://doi.org/10.1109/TNNLS.2017.2755697

32. Liu, Y., Zhang, D., Lu, J., Cao, J.: Global $\mu$-stability criteria for quaternion-valued neural networks with unbounded time-varying delays. Inf. Sci. 360, 273-288 (2016)

33. Shu, H., Song, Q., Liu, Y., Zhao, Z., Alsaadi, F.E.: Global $\mu$-tability of quaternion-valued neural networks with non-differentiable time-varying delays. Neurocomputing 247, 202-212 (2017)

34. Liu, Y., Zhang, D., Lu, J.: Global exponential stability for quaternion-valued recurrent neural networks with time-varying delays. Nonlinear Dyn. 87(1), 553-565 (2017)

35. Chen, X., Li, Z., Song, Q., Hu, J., Tan, Y.: Robust stability analysis of quaternion-valued neural networks with time delays and parameter uncertainties. Neural Netw. 91, 55-65 (2017)

36. Tu, Z., Cao, J., Alsaedi, A., Hayat, T.: Global dissipativity analysis for delayed quaternion-valued neural networks. Neural Netw. 89, 97-104 (2017)

37. Liu, Y., Wang, Z., Liu, X.: Global exponential stability of generalized recurrent neural networks with discrete and distributed delays. Neural Netw. 19(5), 667-675 (2006)

38. Zeng, Z., Huang, T., Zheng, W.X.: Multistability of recurrent neural networks with time-varying delays and the piecewise linear activation function. IEEE Trans. Neural Netw. 21(8), 1371-1377 (2010)

39. Liu, X., Chen, T.: Global exponential stability for complex-valued recurrent neural networks with asynchronous time delays. IEEE Trans. Neural Netw. Learn. Syst. 27(3), 593-606 (2016)

40. Gopalsamy, K.: Leakage delays in BAM. J. Math. Anal. Appl. 325(2), 1117-1132 (2007)

41. Li, X., Fu, X., Balasubramaniam, P., Rakkiyappan, R.: Existence, uniqueness and stability analysis of recurrent neural networks with time delay in the leakage term under impulsive perturbations. Nonlinear Anal., Real World Appl. 11(5), 4092-4108 (2010)

42. Song, Q., Zhao, Z., Li, Y.: Global exponential stability of BAM neural networks with distributed delays and reaction-diffusion terms. Phys. Lett. A 335(2), 213-225 (2005)

43. Zhou, J., Li, S., Yang, Z.: Global exponential stability of Hopfield neural networks with distributed delays. Appl. Math. Model. 33(3), 1513-1520 (2009)

44. Nie, X., Cao, J.: Multistability of competitive neural networks with time-varying and distributed delays. Nonlinear Anal., Real World Appl. 10(2), 928-942 (2009)

45. Chen, X., Song, Q.: Global stability of complex-valued neural networks with both leakage time delay and discrete time delay on time scales. Neurocomputing 121, 254-264 (2013)

46. Song, Q., Zhao, Z:: Stability criterion of complex-valued neural networks with both leakage delay and time-varying delays on time scales. Neurocomputing 171, 179-184 (2016)

47. Boyd, S., Ghaoui, L.E., Feron, E., Balakrishnan, V.: Linear Matrix Inequalities in System and Control Theory. SIAM, Philadelphia (1994)

\section{Submit your manuscript to a SpringerOpen ${ }^{\circ}$ journal and benefit from:}

- Convenient online submission

- Rigorous peer review

- Open access: articles freely available online

- High visibility within the field

Retaining the copyright to your article

Submit your next manuscript at $\gg$ springeropen.com 